\title{
A RESULT FOR SEMI-REGULAR GONTINUED FRACTIONS
}

\author{
P. E. BLANKSBY
}

(Received 8 January 1968)

\section{Introduction}

If $\varphi$ is a real number with $|\varphi|>1$, then a semiregular continued fraction development of $\varphi$ is denoted by

$$
\varphi=\left[a_{1}, a_{2}, a_{3}, \cdots\right]=a_{1}-\frac{1}{a_{2}-} \frac{1}{a_{3}-} \cdots,
$$

where the $a_{i}$ are integers such that $\left|a_{i}\right| \geqq 2$. The expansions arise geometrically by considering the sequence of divided cells of two-dimensional grids (see [1]), and are described by the following algorithm:

$$
\varphi_{n}=a_{n+1}-\frac{1}{\varphi_{n+1}}, \quad\left|a_{n+1}\right| \geqq 2, \quad\left|\varphi_{n+1}\right|>1,
$$

for all $n \geqq 0$, taking $\varphi=\varphi_{0}$. Hence

$$
a_{n+1}=\left[\varphi_{n}\right] \text { or }\left[\varphi_{n}+1\right],
$$

where in this case the square brackets are used to signify the integer-part function. It follows that each irrational $\varphi$ has uncountably many such expansions, none of which has $a_{n}$ constantly equal to 2 (or -2) for large $n$.

Hurwitz [4] has investigated one particular expansion from this uncountable set, namely the one for which

$$
-\frac{1}{2}<\frac{1}{\varphi_{n}} \leqq \frac{1}{2}
$$

for all $n \geqq 0$. This expansion is a development 'to the nearest integer' in an obvious sense. Hurwitz proved that for any such expansion, if we put

then

$$
\theta_{n}=\left[a_{n}, a_{n-1}, \cdots, a_{1}\right]
$$

$$
\begin{aligned}
& \frac{1-\sqrt{ } 5}{2}<\frac{1}{\theta_{n}}<\frac{3-\sqrt{ } 5}{2} \text { when } \varphi_{n}>0, \\
& \frac{\sqrt{ } 5-3}{2}<\frac{1}{\theta_{n}}<\frac{\sqrt{ } 5-1}{2} \text { when } \varphi_{n}<0 . \\
& 145
\end{aligned}
$$


DEFINITION. If $k \geqq 1$ is any real number, we will denote by $E(\varphi, 1 / k)$ that unique semi-regular expansion of $\varphi \geqq k$, for which

$$
\frac{1-k}{k}<\frac{1}{\varphi_{n}} \leqq \frac{1}{k},
$$

and by $E(1 / k)$ the set

$$
E\left(\frac{1}{k}\right)=\left\{E\left(\varphi, \frac{1}{k}\right) \mid \varphi \geqq k, \varphi \text { real }\right\} .
$$

We can detine a pair of minimal intervals $I^{+}(1 / k)$ and $I^{-}(1 / k)$, which are subintervals of $[-1,1]$, and such that for all expansions of $E(1 / k)$ we have

$$
\frac{1}{\theta_{n}} \in\left\{\begin{array}{lll}
I^{+}\left(\frac{1}{k}\right) & \text { when } & \varphi_{n}>0 \\
I^{-}\left(\frac{1}{k}\right) & \text { when } & \varphi_{n}<0 .
\end{array}\right.
$$

Then Hurwitz' result reduces to

$$
\begin{aligned}
& I^{+}\left(\frac{1}{2}\right)=\left(\frac{1-\sqrt{ } 5}{2}, \frac{3-\sqrt{ } 5}{2}\right) \\
& I^{-}\left(\frac{1}{2}\right)=\left(\frac{\sqrt{ } 5-3}{2}, \frac{\sqrt{ } 5-1}{2}\right) .
\end{aligned}
$$

Davenport [3] and others have utilised this result to obtain results on the inhomogeneous minima of indefinite binary quadratic forms. In this case the $I^{ \pm}\left(\frac{1}{2}\right)$ are both of unit length, and so a different type of reduction for quadratic forms may be defined, by relaxing each of the open intervals to a half-open interval, and representing each reduced form by a doubly infinite chain of integers. This gives rise to the notion of a semi-regular expansion of the 'second kind' (see [4]).

It seemed to be of interest to investigate the intervals $I \pm(1 / k)$ for other values of $k \geqq 1$. If we put

$$
L\left(\frac{1}{k}\right)=\max \left\{\text { length of } I^{ \pm}\left(\frac{1}{k}\right)\right\},
$$

then we may pose the following question. For what values of $k$ is $L(1 / k)=1$, and hence after fixing a suitable convention, define a unique semi-regular expansion of the second kind?

In section 3 we will establish the following results. Define the sequences $\left\{\alpha_{r}\right\},\left\{\beta_{r}\right\},\left\{\delta_{r}\right\}$ by

$$
\alpha_{r}=\frac{r}{r+1}, \quad \beta_{r}=\frac{3 r-\sqrt{ }\left(r^{2}+4 r\right)}{2 r}, \quad \delta_{r}=\frac{\sqrt{ }\left(r^{2}+4 r\right)-r}{2} .
$$


THeorem 1. For all $r \geqq 1$,

$$
\begin{aligned}
& I^{+}\left(\alpha_{r}\right)=\left(\beta_{r}-1, \beta_{r}\right) \\
& I^{-}\left(\alpha_{r}\right)=\left(\delta_{r}-1, \delta_{r}\right) \\
& I^{ \pm}\left(\beta_{r}\right)=\left(\beta_{r}-1, \beta_{r}\right) \\
& I^{ \pm}\left(\delta_{r}\right)=\left(\delta_{r}-1, \delta_{r}\right) .
\end{aligned}
$$

Similar results hold for $I^{ \pm}\left(1-\alpha_{r}\right), I^{ \pm}\left(1-\beta_{r}\right), I^{ \pm}\left(1-\delta_{r}\right)$, and these are readily deduced from the theorem.

In section 4 the intervals $I^{ \pm}(1 / k)$ will be discussed for more general $k \geqq 1$. It will be shown that there are uncountably many 'bad' $k$, that is $k$ for which $L(1 / k)>1$. The whole paper could have been presented in the context of binary quadratic forms, the proofs needing only minor modification. I would like to thank the referee for his helpful comments and suggestions on this paper, and to acknowledge the financial support of an 1851 Overseas Scholarship.

\section{Preliminary results}

In this section we will quote a few results for semi-regular continued fractions which we will need in what follows. If $p_{n} / q_{n}$ is the $n^{\text {th }}$ convergent for $\varphi$, that is

then (see [1])

$$
p_{n} / q_{n}=\left[a_{1}, a_{2}, \cdots, a_{n}\right]
$$

$$
\begin{aligned}
p_{0}=1, q_{0} & =0, p_{1}=a_{1}, q_{1}=1, \\
p_{n+1} & =a_{n+1} p_{n}-p_{n-1}, \\
q_{n+1} & =a_{n+1} q_{n}-q_{n-1},
\end{aligned}
$$

and

It follows that

$$
p_{n-1} q_{n}-q_{n-1} p_{n}=1 \quad(n \geqq 1) \text {. }
$$

$$
\varphi_{0}=\left[a_{1}, a_{2}, \cdots, a_{n}, \varphi_{n}\right]=\frac{\varphi_{n} p_{n}-p_{n-1}}{\varphi_{n} q_{n}-q_{n-1}} .
$$

LeMma 1 . If $\varphi=\left[a_{1}, a_{2}, \cdots\right]$ where $a_{j}>0$ for all $j \geqq 1$, then whenever $a_{1}, a_{2}, \cdots, a_{n-1}$ remain constant and $a_{n}$ is increased, the value of $\varphi$ is increased, whatever the values of $a_{n+1}, a_{n+2}, \cdots$. It follows that if $\varphi_{n}$ and $\lambda_{n}$ are also both positive, then

$$
\left[a_{1}, a_{2}, \cdots, a_{n}, \varphi_{n}\right]<\left[a_{1}, a_{2}, \cdots, a_{n},-\lambda_{n}\right] .
$$

The proof may be found in [1].

LEMma 2. We have, if $a_{1}>0$,

$$
\varphi<\left[a_{1}, a_{2}, \cdots, a_{n}, \varphi\right]
$$


if and only if

$$
\varphi<\left[\overline{a_{1}, \cdots, a_{n}}\right]
$$

where, as usual, the upper bar is used to denote a periodic expansion.

This result follows from (4).

The repetition of a finite sequence of partial quotients will be denoted by a suitable subscript, and we will incorporate the convention that a zero subscript means that the corresponding segment be deleted from the chain. We will use round external brackets to denote an ordinary continued fraction development.

Lemma 3.

$$
\left[2_{r}, \varphi^{\prime}\right]=\frac{(r+1) \varphi^{\prime}-r}{r \varphi^{\prime}-(r-1)}, \text { for } \quad r \geqq 0
$$

If in ordinary continued fractions

$$
\varphi=\left(a, r+1, \varphi^{\prime}\right), a \geqq 0, \varphi^{\prime}>1,
$$

then in semi-regular continued fractions

$$
\varphi+1=\left[a+2,2_{r}, \varphi^{\prime}+1\right] .
$$

The proof is straight forward and may be found in [2]. This relationship enables any expansion from the set $E(1)$ to be transformed into the corresponding ordinary continued fraction expansion. Using the above conventions, and inserting an appropriate $2_{0}$ into the semi-regular expansion, if necessary, we have for $a_{i}>0$,

$$
\begin{aligned}
\varphi & =\left(a_{1}, a_{2}, a_{3}, a_{4}, \cdots\right) \text { if and only if } \\
\varphi+1 & =\left[a_{1}+2,2_{a_{2}-1}, a_{3}+2,2_{a_{4}-1}, \cdots\right] .
\end{aligned}
$$

It is clear that for $k>1, E(\mathrm{I} / k)$ contains no expansion with a partial quotient equal to \pm 1 , for if say

$$
\varphi_{n}=1-\frac{1}{\varphi_{n+1}}, \quad \text { with } \quad \frac{1-k}{k}<\frac{1}{\varphi_{n+1}}<0,
$$

then $\varphi_{n} \geqq k$ implies that

$$
k \leqq \varphi_{n}<1-\frac{1-k}{k}=2-\frac{1}{k},
$$

a contradiction. 


\section{Proof of Theorem 1}

We first note that after using (4)

$$
\left\{\begin{array}{l}
1 / \alpha_{r}=\left[2_{r}\right], \\
1 / \beta_{r}=\left[\overline{2_{r-1}, 3}\right], \\
1 / \delta_{r}=\left[2, \overline{2_{r-1}, 3}\right] .
\end{array}\right.
$$

It then follows from Lemma 2 that

$$
\cdots<\beta_{r-1}<\alpha_{r-1}<\delta_{r-1}<\beta_{r}<\alpha_{r}<\delta_{r}<\cdots
$$

We also have

$$
\left\{\begin{array}{l}
1 /\left(1-\alpha_{r}\right)=r+1, \\
1 /\left(1-\beta_{r}\right)=[r+1, \overline{r+2}], \\
1 /\left(1-\delta_{r}\right)=[\overline{r+2}] .
\end{array}\right.
$$

These expansions may be obtained by an application of the transformation (5), as the following example demonstrates.

hence by (5)

$$
\frac{1}{\beta_{r}}=\left[2,2_{r-2}, \overline{3,2_{r-1}}\right]
$$

$$
\frac{1}{\beta_{r}}-1=(0, r-1, \overline{1, r}),
$$

and

$$
\frac{\beta_{r}}{1-\beta_{r}}=(r-1, \overline{1, r})
$$

Thus, by (5) again, we obtain

$$
\frac{\beta_{r}}{1-\beta_{r}}+1=\frac{1}{1-\beta_{r}}=\left[r+1, \overline{2_{0}, r+2}\right]=[r+1, \overline{r+2}] .
$$

Consider the set of expansions $E\left(\alpha_{r}\right)$. We cannot have for any $n$,

else

$$
\varphi_{n}=\left[2_{r}, \varphi_{n+r}\right], \text { where } \varphi_{n+r}>0,
$$

$$
\varphi_{n}=\frac{(r+1) \varphi_{n+r}-r}{r \varphi_{n+r}-(r-1)}<\frac{r+1}{r},
$$

which contradicts that the expansion belongs to $E\left(\alpha_{r}\right)$. However, if

$$
\varphi_{n}=\left[2_{r-1}, \varphi_{n+r-1}\right],
$$

where $\varphi_{n+r-1}>2$, then $\varphi_{n}>(r+1) / r$, as required, Hence, whenever $a_{m+1}>0, a_{m}>0$, we have by (6) and Lemma 1, 


$$
\theta_{m}>\left[\overline{2_{r-1}, 3}\right]=1 / \beta_{r} \text {. }
$$

Now if for some $n$

$$
\varphi_{n}=\left[2_{r+1}, \varphi_{n+r+1}\right],
$$

where $\varphi_{n+r+1}<-2$, then $\varphi_{n}<(r+1) / r$, but if

$$
\varphi_{n}=\left[2_{r}, \varphi_{n+r}\right],
$$

where $\varphi_{n+r}<0$, then $\varphi_{n}>(r+1) / r$. Hence it follows from (6) and Lemma 1, that whenever $a_{m+1}<0, a_{m}>0$, that

$$
\theta_{m}>\left[2, \overline{2_{r-1}, 3}\right]=1 / \delta_{r} .
$$

If for some expansion from the set $E\left(\alpha_{r}\right)$ we have

$$
\varphi_{n}=\left[-r-1, \varphi_{n+1}\right] \text {, }
$$

then we conclude that $\varphi_{n+1}>0$, and clearly $r+1$ is the smallest absolute value permissible for negative partial quotients. Consequently, by (8) and Lemma 1, we have for all $m$ for which $\varphi_{m}>0$, and $a_{m}<0$,

$$
\left|\theta_{m}\right|>[r+1, \overline{r+2}]=1 /\left(1-\beta_{r}\right),
$$

and for all $m$ for which $\varphi_{m}<0$, and $a_{m}<0$,

$$
\left|\theta_{m}\right|>[\overline{r+2}]=1 /\left(1-\delta_{r}\right) .
$$

Thus we have shown that

$$
\begin{aligned}
& I^{+}\left(\alpha_{r}\right)=\left(\beta_{r}-1, \beta_{r}\right), \\
& I^{-}\left(\alpha_{r}\right)=\left(\delta_{r}-1, \delta_{r}\right) .
\end{aligned}
$$

Let us now consider the expansions from the set $E\left(\beta_{r}\right)$. It is again clear that we cannot have for any $n$

$$
\varphi_{n}=\left[\boldsymbol{2}_{r}, \varphi_{n+r}\right], \text { where } \varphi_{n+r}>0,
$$

but, by Lemma 1, we may have

$$
\varphi_{n}=\left[\mathbf{2}_{r-1}, \varphi_{n+\boldsymbol{r}-1}\right],
$$

provided $\varphi_{n+r-1} \geqq\left[3,1 / \beta_{r}\right]$. In the case when

$$
\varphi_{n}=\left[\boldsymbol{2}_{r}, \varphi_{n+r}\right], \text { where } \varphi_{n+r}<0,
$$

then by hypothesis

$$
1 /\left|\varphi_{n+r}\right|<1-\beta_{r},
$$

and so by (6) and Lemma 1 ,

$$
\varphi_{n}=\left[2_{r-1}, 2+\frac{1}{\left|\varphi_{n+r}\right|}\right]<\left[2_{r-1}, 3-\beta_{r}\right]=1 / \beta_{r} .
$$


It therefore follows that whatever the sign of $\varphi_{m}$, we have for $a_{m}>0$,

$$
\theta_{m}>\left[\overline{2_{r-1}, 3}\right]=1 / \beta_{r} .
$$

Now suppose that for some $n$ we have

then clearly $\varphi_{n+1}>0$, and

$$
\varphi_{n}=\left[-r, \varphi_{n+1}\right]
$$

$$
\begin{aligned}
\left|\varphi_{n}\right| & =r+\frac{1}{\left|\varphi_{n+1}\right|} \\
& \leqq r+\beta_{r} \\
& =\left[r+1,\left(1-\beta_{r}\right)^{-1}\right] \\
& <1 /\left(1-\beta_{r}\right),
\end{aligned}
$$

if and only if $1 /\left(1-\beta_{r}\right)>[\overline{r+1}]$, by Lemma 2 , and by (8) this condition is clearly satisfied, leading to a contradiction. Now

$$
\varphi_{n}=\left[-r-1, \varphi_{n+1}\right]
$$

is a permissible expansion, whatever the sign of $\varphi_{n+1}$, provided it is large enough. However we cannot have

$$
\varphi_{n}=\left[-r-1,-r-1, \varphi_{n+2}\right],
$$

when $\varphi_{n+2}<0$, since this implies

$$
\left|\varphi_{n}\right|<1 /\left(1-\beta_{r}\right) .
$$

If $\varphi_{n+2}>0$, then $\varphi_{n+2} \geqq 1 / \beta_{r}$, and

$$
\begin{aligned}
\left|\varphi_{n}\right| & =\left[r+1, r+1+\frac{1}{\varphi_{n+2}}\right] \\
& \leqq\left[r+1, r+2,\left(1-\beta_{r}\right)^{-1}\right] \\
& <1 /\left(1-\beta_{r}\right),
\end{aligned}
$$

by (8) and Lemma 2 , since $1 /\left(1-\beta_{r}\right)>[\overline{r+1, r+2}]$.

Hence it follows readily that if $a_{m}<0$,

Consequently

$$
\left|\theta_{m}\right|>[r+1, \overline{r+2}]=1 / 1-\beta_{r} .
$$

$$
I^{ \pm}\left(\beta_{r}\right)=\left(\beta_{r}-1, \beta_{r}\right) .
$$

The result for $I^{ \pm}\left(\delta_{r}\right)$ follows analogously.

On examining the proofs for the preceding results, we notice that they remain valid if we replace (3) by

$$
\frac{1-k}{k} \leqq \frac{1}{\varphi_{n}}<\frac{1}{k}
$$$$
(n \geqq 0) \text {, }
$$ 
the only modification required being the occasional interchange of $<$ and $\leqq$. Denote this set of expansions by $E^{\prime}(1 / k)$. Then the set obtained by negating all the expansions of $E^{\prime}(1 / k)$ is just the set $E(1-1 / k)$. It follows from Theorem 1 that

$$
\begin{aligned}
& I^{+}\left(1-\alpha_{r}\right)=\left(-\delta_{r}, 1-\delta_{r}\right) \\
& I^{-}\left(1-\alpha_{r}\right)=\left(-\beta_{r}, 1-\beta_{r}\right) \\
& I^{ \pm}\left(1-\beta_{r}\right)=\left(-\beta_{r}, 1-\beta_{r}\right) \\
& I^{ \pm}\left(1-\delta_{r}\right)=\left(-\delta_{r}, 1-\delta_{r}\right) .
\end{aligned}
$$

\section{Further results}

In this section we prove the following theorem.

ThEOREM 2. For any $k$ satisfying either

$$
\alpha_{r-1}<\frac{1}{k}<\delta_{r-1}, \quad \text { or } \quad \beta_{r}<\frac{1}{k}<\alpha_{r},
$$

we have

$$
L\left(\frac{1}{k}\right)>1 .
$$

Proof. In the first case (6) and Lemma 1 imply that

hence

$$
k=\left[2_{r-1}, a, \cdots\right], \quad \text { where } \quad a \geqq 3 ;
$$

$$
\varphi_{n}=\left[2_{r-1}, a_{n+r}, \cdots\right] \text {, where } a_{n+r}>0
$$

satisfies $\varphi_{n}>k$ provided $a_{n+r}$ is large enough. Now put

$$
\varphi=r+\frac{1}{k}=\left[r+1, \frac{k}{k-1}\right],
$$

then by Lemma $2, \varphi>k /(k-1)$ provided

$$
\frac{k}{k-1}<[\overline{r+1}]=1 /\left(1-\delta_{r-1}\right),
$$

which is satisfied, by the hypothesis.

Now let

$$
\gamma=p-\frac{k}{k-1}>0
$$

If we have for some $k^{*}>k, \varphi_{n}=\left[-r, k^{*}\right]$, then 


$$
\begin{aligned}
\left|\varphi_{n}\right|=r+\frac{1}{k^{*}} & =\varphi+\frac{1}{k^{*}}-\frac{1}{k} \\
& =\frac{k}{k-1}+\gamma+\frac{1}{k^{*}}-\frac{1}{k} \\
& >\frac{k}{k-1},
\end{aligned}
$$

provided $k^{*}$ is sufficiently close to $k$. Choose $k^{*}$ to be rational (and if $k$ is rational, take $k^{*}=k$ ), and close enough to $k$. Expanding $k^{*}$ by (3), we obtain the finite expansion $E\left(k^{*}, 1 / k\right)$, say,

$$
k^{*}=\left[2_{r-1}, b_{1}, \cdots, b_{n}\right] \text {, where } b_{1}>0 .
$$

Consider the chain

$$
\left[a_{1}, \cdots, a_{t},-r, 2_{r-1}, b_{1}, \cdots, b_{n}, b, c, 2_{r-1}, b, \cdots\right],
$$

with $a_{t}<0$ and $c>0$. It is clear that whether $k$ is rational or irrational, $\left[b_{j}, \cdots, b_{n}\right]$ cannot equal either $k$ or $k /(1-k)$ for any $j(1 \leqq j \leqq n)$, and so, provided $b$ is sufficiently large and of the correct sign, the chain belongs to the set of expansions $E(1 / k)$, for all large $a_{1}, \cdots, a_{t-1}$. Hence we have for the corresponding subscripts $p$ and $m$,

$$
\begin{aligned}
\left|\theta_{p}\right| & =\left[r,\left|a_{t}\right|, \cdots,-a_{1}\right]<r, \varphi_{p}>0, \\
\theta_{m} & =\left[2_{r-1}, c, \cdots, a_{1}\right]<\left[2_{r-1}\right]=r /(r-1), \varphi_{m}>0 .
\end{aligned}
$$

Thus the length of $I^{+}(1 / k)$ exceeds unity. Since the $a_{1}, \cdots, a_{t-1}$, are arbitrary, then there are chains in which there are infinitely many pais $p, m$, for which (10) holds.

In the second case, it may be checked readily by methods similar to the above that

$$
k=\left[2_{r}, x\right] \text {, where } x<0 .
$$

It can be verified that the following expansion belongs to the set $E(1 / k)$, provided that $b$ is large enough, and the $a_{1}, \cdots, a_{p}$ are chosen judiciously.

$$
\left[a_{1}, \cdots, a_{p},-r-1, b, c, 2_{r}, x\right] \text {, }
$$

where $a_{p}<0, b<0$, and $c>0$. Thus for some $m$ and $t$ we have

$$
\begin{aligned}
\left|\theta_{m}\right| & =\left[r+1,\left|a_{p}\right|, \cdots,-a_{1}\right]<r+1, & & \varphi_{m}<0, \\
\theta_{t} & =\left[2_{r}, c, \cdots, a_{1}\right]<\left[2_{r}\right]=(r+1) / r, & & \varphi_{t}<0 .
\end{aligned}
$$

Hence the length of $I^{-}(1 / k)$ exceeds unity.

By methods similar to those used to obtain (9), we may prove analogous results for sequences of intervals in $\left[0, \frac{\mathbf{1}}{\mathbf{2}}\right]$. 
The question of the values taken by $L(1 / k)$ when $\delta_{r-1}<1 / k<\beta_{r}$ has a more complicated answer, and we will not investigate it here. There are sequences of values of $k$ in this range for which $L(1 / k)=1$. For example

$$
\begin{aligned}
k_{r, m} & =\left[2_{r-1}, 3, \overline{\left(2_{r-1}, 3\right)_{m}, 2_{r-2}, 3}\right] \\
k_{r, m, p} & =\left[\left(2_{r-1}, 3\right)_{m}, \overline{2_{r-1}, 3,\left(2_{r-2}, 3,\left(2_{r-1}, 3\right)_{m}\right)_{p}}\right] .
\end{aligned}
$$

There are also sequences of intervals for which $L(1 / k)>1$. It seems reasonable to conjecture that the set of $k$ for which $L(1 / k)=1$, has measure zero.

\section{References}

[1] Barnes, E. S. and Swinnerton-Dyer, H. P. F., The inhomogeneous minima of binary quadratic forms III, Acta Math. 92 (1954), 199-234.

[2] Blanksby, P. E., A restricted inhomogeneous minimum for forms, to appear $J$. Aust. Math. Soc.

[3] Davenport, H., Indefinite binary quadratic forms, and Euclid's algorithm in real quadratic fields, Proc. London Math. Soc. (2) 53 (1951), 65-82.

[4] Hurwitz, A., Math. Werke II, 84-115.

\section{Trinity College}

Cambridge 